\title{
Analysis of Volatile Components From the Extracts of Honeysuckle by Microbial Fermentation
}

\author{
Wei Min, Chen Shuo, Song Xu-yan*, Li Ran, Pan Xi, Guo Guo-ning \\ Technology Center of China Tobacco Hubei Industry Limited-liability Company, Wuhan, China
}

Email address:

weimin@hbtobacco.cn (Wei Min), 99486535@qq.com (Song Xu-yan)

${ }^{*}$ Corresponding author

\section{To cite this article:}

Wei Min, Chen Shuo, Song Xu-yan, Li Ran, Pan Xi, Guo Guo-ning. Analysis of Volatile Components From the Extracts of Honeysuckle by Microbial Fermentation. Science Discovery. Vol. 6, No. 1, 2018, pp. 43-46. doi: 10.11648/j.sd.20180601.17

Received: March 1, 2018; Accepted: April 17, 2018; Published: May 23, 2018

\begin{abstract}
In order to analysis the volatile components of honeysuckle extract, the honeysuckle extracts by Raoultella planticola fermented and its contrast extracts were analyzed by GC/MS after simultaneous distillation extraction. The results showed: In compared with the contrast, (1) 35volatile components were identified from the fermented honeysuckle extract, including 22 kinds of new: 3- hydroxy -2- methyl ethyl ketone, hexanal, isoamyl propionate, benzene ethanol, two hydrogen actinidiolide, etc.,which accounted for more than $40 \%$ of the total relative percentage content; (2) And 23 kinds of volatile components' content changed, including linalool, $\alpha$-terpineol increased, at the same time, the stimulus components disappeared, such as dibutyl phthalate. After fermentation, the volatile components of honeysuckle extract has changed in a big way, with fragrance, fruit sweet, creamy and rich aroma, and stimulating ingredients reduce, leading to improve the quality of aroma.
\end{abstract}

Keywords: Enterobacter Mori, Biotransformation, Honeysuckle Extract, GC/MS, Volatile Components

\section{微生物发酵金银花提取物的挥发性成分分析}

魏敏, 陈硕, 宋旭艳*, 李冉, 潘䂀, 郭国宁

湖北中烟工业有限责任公司技术中心, 武汉, 中国

\section{邮箱}

weimin@hbtobacco.cn（魏敏），99486535@qq.com（宋旭艳）

摘要：采用桑肠杆菌(Enterobacter mori)发酵金银花提取物，经同时蒸馏萃取，气相质谱对比分析发酵金银花提取物及 其对照的挥发性成分。结果表明, 与对照相比较, (1) 微生物发酵金银花提取物鉴定出36种挥发性成分, 新增有23种, 主要包括3-差基-2-丁酮、己醛、丙酸异成酯、苯乙醇、二氢狝猴桃内酯等香气成分，占相对百分含量 $40 \%$ 以；(2)相 对含量发生变化的有 13 种，芳樟醇、 $\alpha$-松油醇等大幅增加; 邻苯二甲酸二正丁酯等刺激性成分消失。微生物发酵后金 银花提取物挥发性香气成分改变大，带有清香、水果甜香、奶香等丰富香气，刺激性成分减少，香气质提高。

关键词: 桑肠杆菌, 微生物转化, 金银花提取物, GC/MS, 挥发性成分 


\section{1. 引言}

金银花为忍冬科植物忍冬Lonicera japonica Thunb. 的 干燥花蕾或带初开的花 [1], 主要含有绿原酸、木犀草 苷等成分, 其化学组成复杂, 数目繁多, 并且在抑菌、疏 散风热等方面也起着显著作用, 是金银花的有效部位之一 $[2,3]$ 。金银花在抗病毒活性方面不仅受到人们的关注 而且也得到了广泛的应用 [4] ; 在各种因素对挥发性化 学成分影响方面也有不少研究报道, 如烘干 [5]、不同 产地 $[6]$ 、不同部位 $[3]$ 、变种 $[7]$ 、乳酸菌发酵 $[8]$ 等; 金银花致香成分检测及在烟草中的应用也做了相关研 究 $[9,10]$ 。本工作拟利用产香菌桑肠杆菌Enterobacter mori 发酵金银花提取物, 采用同时蒸馏萃取和气相色谱-质谱 (GC/MS) 检测分析其挥发性成分, 以研究微生物发酵对 金银花提取物香气成分的改善, 为这一药食两用资源的开 发利用提供试验科学依据。

\section{2. 材料与方法}

\section{1. 材料和仪器}

桑肠杆菌Enterobacter mori VL4-3 CCTCC M2012020, 从香荚兰植物的茎中篮选得到, 于 2012 年1月16日保藏于 武汉大学中国典型培养物保藏中心;

斜面培养基 (马铃薯葡萄糖琼脂培养基, 北京三药科 技开发公司, BR）：马铃薯浸液 $180 \mathrm{~mL} / \mathrm{L}$, 葡萄糖 $18 \mathrm{~g} / \mathrm{L}$, 琼脂 $14 \mathrm{~g} / \mathrm{L}$; 原始种子培养基(麦芽糖蛋白胨培养基): 麦 芽糖 $50 \mathrm{~g} / \mathrm{L}$, 蛋白胨 $9 \mathrm{~g} / \mathrm{L}$, 氯化钠 $4.5 \mathrm{~g} / \mathrm{L}$; 葡萄糖、琼脂、 麦芽糖、蛋白胨、氯化钠、二氯甲烷，无水硫酸钠 (国药 集团化学试剂有限公司, AR），95\%乙醇（食用级）; 发 酵底物: 市售金银花（产地湖北）粉粹到 40 目后，回流提 取3小时, 抽滤后的滤液。

生化培养箱( 上海精宏实验设备有限公司, SHP-080)、 灭菌锅 (上海三申医疗器械有限公司, YX.350Z) 、超净 工作台 (苏净集团安泰公司, SW-CJ-1FD)、恒温摇床 (武 汉中科科仪技术发展有限责任公司, HQL 300B）、气相 色谱质谱联用仪（安捷伦科技公司, Agilent GC:6890N MS:5973)。

\section{2. 方法}

\subsection{1. 微生物发酵金银花提取物的制备}

将金银花和水按质量比1: 40混合, 回流提取 $3 \mathrm{~h}$ 后, 抽滤, 取滤液, $115^{\circ} \mathrm{C}$ 高压灭菌25分钟。

将桑肠杆菌L4-3接种到马铃薯葡萄糖琼脂斜面培养 基上, 在恒温培养箱中 $31^{\circ} \mathrm{C} 、 \mathrm{pH}$ 自然, 静置培养 $48 \mathrm{~h}$ 后置 于 $4{ }^{\circ} \mathrm{C}$ 冰箱中; 用上述斜面培养物, 接种到种子培养基, 在 $31^{\circ} \mathrm{C}$, 转速 $130 \mathrm{rpm}$ 条件下振荡培养 $24 \mathrm{~h}$, 转接 1 次后制 得种子培养液; 将种子培养液按体积比 $18 \%$ 接种到金银花 滤液中, 装罐量为 $20 \%$, 在 $\mathrm{pH}$ 自然, 温度 $32^{\circ} \mathrm{C}$, 转速 $120 \mathrm{rpm}$, 不避光条件下, 发酵 $36 \mathrm{~h}$, 得到金银花发酵液, 抽滤浓缩 得到相对密度约为 1.3 的浸毫, 与 $95 \%$ 乙醇按质量比 $1: 3$ 进行醇沉, 过夜后抽滤, 除去杂质保留滤液, 并减压浓缩
除去溶剂, 制得黄褐色浸膏, 密度为 1.11 , 为微生物发酵 金银花提取物。

\subsection{2. 挥发性成分同时蒸馏萃取}

将 $5 \mathrm{~g}$ 微生物发酵金银花提取物装入圆底烧瓶中, 加入 $400 \mathrm{~mL}$ 水, 用电热套加热至沸腾, 另一侧的圆底烧瓶中加 入 $200 \mathrm{~mL}$ 的二氯甲烷, $60^{\circ} \mathrm{C}$ 水浴沸腾, 待中间分液管处液 面出现分层后计时萃取 $2.5 \mathrm{~h}$ 。萃取结束后, 向二氯甲烷萃 取液中加入无水硫酸钠, 过夜, 过滤并浓缩至 $1 \mathrm{~mL}$, 浓缩 液用于进行 GC/MS分析。同样的方法进行 $5 \mathrm{~g}$ 未发酵金银 花提取物, 同时蒸馏萃取后作为对照样, 进行 GC/MS 分析。

\subsubsection{GC/MS对比检测分析}

HP-5MS(50 m $\times 0.25 \mathrm{~mm} \times 0.25 \mu \mathrm{m})$ 毛细管柱; 进样温 度: $250^{\circ} \mathrm{C}$; 分流比 $25: 1$; 载气: $\mathrm{He}, 1 \mathrm{~mL} / \mathrm{min}$; 升温程 序: 从 $50^{\circ} \mathrm{C}(1 \mathrm{~min})$ 以 $5^{\circ} \mathrm{C} / \mathrm{min}$ 升到 $250^{\circ} \mathrm{C}(5 \mathrm{~min})$; 传 输线温度: $250^{\circ} \mathrm{C}$; 离子源: $\mathrm{EI}$ 源; 电子能量: $70 \mathrm{eV}$; 扫 描范围: 50 400 amu; 使用WILEY和MINILAB谱库进行 检索。

\section{3. 结果与分析}

微生物发酵金银花提取物及其对照的挥发性香气成 分GC/MS分析结果（表1）。结果表明：(1)金银花提取物 对照共分离鉴定出 23 种挥发性成分, 总相对百分含量为 $57.05 \%$, 包括酯类9种 $(32.00 \%)$, 酸类6种 $(22.67 \%)$, 醇类3种 $(0.93 \%)$, 酮类 2 种 $(0.77 \%)$, 酚类1种 $(0.40 \%)$, 其他杂环类2种; (2)微生物发酵金银花提取物共分离鉴定 出36种挥发性成分, 总相对百分含量为 $77.38 \%$, 包括酯类 10 种 $(23.61 \%)$, 酸类4种 ( $13.53 \%)$, 酮类3种 ( $21.58 \%)$, 醛类3种 $(7.19 \%)$, 醇类 3 种 $(3.50 \%)$, 烯类 2 种 $(0.30 \%)$, 酚类 2 种 $(0.15 \%)$ ，烷烃 1 种 $(0.70 \%)$, 其他杂环类化合 物 8 种, 其中新出现了 23 种成分, 主要有3-羟基-2-丁酮、 己醛、丙酸异戊酯、3-糠醛、二甲基呋喃、苯乙醇、二氢 猕猴桃内酯等, 占总挥发性成分相对百分含量 $40 \%$ 以上。 其中, 3-羟基-2-丁酮 (21.42\%), 具有强烈的奶油、脂肪、 白脱样香气, 高度稀释后有令人愉快的奶香气; 己醛, 呈 生的油脂和青草气及苹果香味; 丙酸异戊酯, 具有水果香 味, 似杏、悬钩子、凤梨洋气息; 苯乙醇, 具有柔和、愉 快而持久的玫瑰香气; 二氢猕猴桃内酯 $(7.37 \%)$, 带有 香豆素样香气, 并有廂香样气息; 还有3-糠醛、二甲基呋 喃、五甲基环戊烯、2,3-二氢-3,3-二甲基-4-硝基苯并呋喃 -2-酮、红没药醇氧化物 A， 9,12 -十八碳二烯酸甲酯、2甲基-7-苯基吲哚、1-甲基-2-苯基吲哚、已二酸二异辛酯、 正十八烷, 邻苯二甲酸二异辛酯等。(3)芳樟醇, 具有铃兰 香气, $\alpha$-松油醇, 具有优雅的紫丁香香气; 以及肉豆冦酸、 十六酸乙酯等6种香气成分相对百分含量有不同程度的提 高。相对百分含量降低的有2,6-二叔丁基-4-甲基苯酚, 为 刺激性成分; 棕榈酸, 可能被转化分解为其他小分子香气 物质。(4)经微生物发酵后, 金银花提取液中一些成分消失, 包括领苯二甲酸二乙酯、领苯二甲酸二正丁酯等刺激性成 分, 占对照相对百分含量的 $12.95 \%$, 以及其他一些香气成 
分, 可能经转化、分解为其他小分子挥发性物质。所有这 果甜香、奶香等丰富香气, 刺激性成分减少, 香气质提高。 些香气糅合在一起赋予微生物发酵金银花提取物清香、水

表1 微生物发酵金银花提取物与其对照的挥发性成分 GC/MS对比分析。

\begin{tabular}{|c|c|c|c|c|c|}
\hline \multirow{2}{*}{ 峰号 } & \multicolumn{2}{|c|}{ 保留时间RT(min) } & \multirow{2}{*}{ 化合物 } & \multicolumn{2}{|c|}{ 相对百分含量（\%) } \\
\hline & 对照 & 发酵金银花提取物 & & 对照 & 发酵金银花提取物 \\
\hline 1 & - & $5.62-$ & 3-（4-硝基苯氨基）-吲哚 & - & 0.58 \\
\hline 2 & - & 6.26 & 3-羟基-2-丁酮 & - & 21.42 \\
\hline 3 & - & 8.56 & 己醛 & - & 2.27 \\
\hline 4 & - & 9.27 & 丙酸异戊酯 & - & 1.35 \\
\hline 5 & 9.51 & - & 异戊酸 & 0.18 & - \\
\hline 6 & - & 9.75 & 3-糠醛 & - & 4.83 \\
\hline 7 & - & 9.81 & 二甲基呋喃 & - & 2.94 \\
\hline 8 & - & 12.33 & 五甲基环戊烯 & - & 0.15 \\
\hline 9 & 14.05 & 14.12 & 4-乙烯基吡啶 & 0.13 & 0.13 \\
\hline 10 & 18.06 & 18.11 & 芳樟醇 & 0.62 & 1.78 \\
\hline 11 & - & 19.10 & 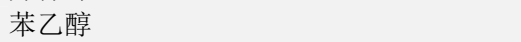 & - & 1.22 \\
\hline 12 & 20.49 & - & 2,2,6-三甲基-6-乙烯基四氢-2H-呋喃-3-醇 & 0.10 & - \\
\hline 13 & 21.08 & 21.14 & $\alpha$-松油醇 & 0.21 & 0.50 \\
\hline 14 & - & 21.99 & $\alpha$-4-二甲基-3 -环己烯-1乙醛 & - & 0.09 \\
\hline 15 & 25.58 & 25.61 & 丙酸苯乙酯(内标) & 3.44 & 3.48 \\
\hline 16 & 26.62 & - & 大马酮- & 0.16 & - \\
\hline 17 & - & 29.66 & 2,4-二叔丁基酚 & - & 0.08 \\
\hline 18 & 29.78 & 29.84 & 2,6-二叔丁基-4-甲基苯酚 & 0.40 & 0.07 \\
\hline 19 & 30.15 & 30.19 & 六甲基-1,3 -环己二烯 & 0.15 & 0.15 \\
\hline 20 & - & 30.72 & 二氢猕猴桃内酯 & - & 7.38 \\
\hline 21 & 31.77 & - & 邻苯二甲酸二乙酯 & 1.02 & - \\
\hline 22 & - & 33.46 & 红没药醇氧化物 A & - & 0.51 \\
\hline 23 & 34.08 & - & 甲基庚烯酮 & 0.61 & - \\
\hline 24 & 35.18 & 35.23 & 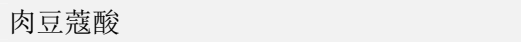 & 0.83 & 1.40 \\
\hline 25 & 37.71 & 37.79 & 邻苯二甲酸丁基酯2-乙基己基酯 & 4.87 & 5.29 \\
\hline 26 & 38.68 & 38.74 & 邻苯二甲酸, 6-乙基-3-辛基丁基酯 & 0.62 & 1.15 \\
\hline 27 & - & 39.05 & 2,3-二氢-3,3-二甲基-4-硝基苯并呋喃-2-酮 & - & 0.16 \\
\hline 28 & 39.28 & 39.39 & 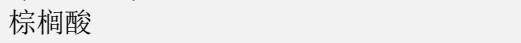 & 15.75 & 9.23 \\
\hline 29 & 39.68 & - & 邻苯二甲酸二正丁酯 & 11.93 & - \\
\hline 30 & 39.95 & 40.02 & 十六酸乙酯 & 0.94 & 1.69 \\
\hline 31 & - & 42.04 & 9,12 -十八碳二烯酸甲酯 $r$ & - & 0.46 \\
\hline 32 & - & 42.12 & 2-甲基-7-苯基吲哚 & - & 0.20 \\
\hline 33 & - & 42.55 & 1-甲基-2-苯基吲哚 & - & 0.74 \\
\hline 34 & 42.70 & 42.75 & 亚油酸 & 1.93 & 0.84 \\
\hline 35 & 42.76 & 42.82 & 十八碳-9-烯酸 & 2.52 & 2.06 \\
\hline 36 & 43.27 & 43.33 & 亚油酸乙酯 & 1.74 & 1.47 \\
\hline 37 & - & 43.89 & 十五烷酸-2,6,10,14-四甲基-甲酯 & - & 0.42 \\
\hline 38 & - & 43.96 & 柠檬酸丁酯 & - & 0.52 \\
\hline 39 & 48.53 & - & 脱氢枞酸甲酯 & 0.33 & - \\
\hline 40 & - & 49.36 & 己二酸二异辛酯 ～～～～～～ & - & 1.99 \\
\hline 41 & - & 50.59 & 2,2'-亚甲基双-(4-甲基-6-叔丁基苯酚) & - & 1.09 \\
\hline 42 & - & 50.98 & 2-苯基-1一苯并吡喃 & - & 0.77 \\
\hline 43 & 51.77 & - & 脱氢枞酸 & 1.46 & - \\
\hline 44 & - & 52.83 & 正十八烷 & - & 0.70 \\
\hline 45 & 55.39 & - & 邻苯二甲酸单乙基己基酯 & 9.94 & - \\
\hline 46 & - & 55.41 & 邻苯二甲酸二异辛酯 & - & 2.35 \\
\hline 47 & 57.89 & - & 7一氧脱氢枞酸甲基酯 & 0.61 & - \\
\hline 48 & - & 63.32 & 二甲基一苯并 $[\mathrm{h}]$ 喹啉 & - & 0.17 \\
\hline
\end{tabular}

注: (1)符号“一”表示未检出; (2)内标为丙酸苯乙酯。

\section{4. 结论}

经桑肠杆菌（Enterobacter mori）发酵后，金银花提 取物挥发性香气成分发生了较大的改变, 相对百分含量总 量从 $57.05 \%$ 提高到 $77.38 \%$, 成分、种类增多, 香气更丰富 自然, 带有清香、水果甜香、奶香等丰富香气, 同时刺激
性成分减少, 香气质提高。微生物在增殖过程中可产生庞 大的高活性酶系, 如多糖水解酶类、蛋白酶类、酯化酶类、 氧化还原酶类及裂合酶类等, 在酶促作用、化学作用及微 生物体内复杂代谢的协同作用下, 以金银花提取液为原料 发生分解、降解、氧化、还原、聚合、偶联、转化等作用, 形成复杂的低分子化合物, 其中包括各种香味化合物, 如 醇类、醛类、酮类、酸类、酯类、酚类、吡喃类等。本论 
文期望能对金银花这一药食两用资源的研究开发提供试 验科学依据, 并对微生物发酵技术开拓金银花提取物的资 源利用方面提供参考。在此研究基础上, 希望对微生物发 酵金银花香料的生产工艺进一步优化, 并对其应用进行研 究。

\section{参考文献}

[1] 国家药典委员会.中华人民共和国药典, 四部[S].北京:中国 医药科技出版社，2015:203。

[2] 管仁伟,王亮,曲永胜,等.“九丰一号”金银花挥发性成分的 GC-MS分析[J].中成药，2014，36(11):2367-2371。

[3] 杨俊杰, 陈琼。河南野生忍冬叶、花、果挥发性成分比较 $[\mathrm{J}]$ 。福建林业科技,2015,42(2):5-8。

[4] 王亚琼, 陈卫, 钟水生, 等。金银花清热解毒作用的血清 代谢组学研究 [J].中药材, 2016,39(5):1129-1133。
[5] 杨金平, 高玲, 管仁伟, 等。烘干对金银花挥发性成分的 影响[J].时珍国医国药, 2016,27(4):869-871。

[6] 杜成智, 冯旭, 王卉, 等。不同产地金银花挥发性成分的 GC-MS 分析[J].江苏农业科学,2014,42(7):313-315。

[7] 崔婷婷, 单长松, 吴澎, 等。金银花和红银花挥发性成分 的顶空固相微萃取气质联用检测与比较 [J]. 园艺学报, 2015 , 42(11):2283-2290。

[8] 陈学红, 秦卫东, 马利, 等。乳酸菌发酵对金银花抗氧化 活性的影响[J].农业机械，2013，26(9):84-87。

[9] 景延秋, 全琳, 罗辉, 等。金银花中挥发性及半挥发性成 分的测定及其在烟草中的应用展望 [J]. 江西农业学 报,2010,22(12):120-123。

[10] 解民, 张琳。金银花萃取物致香成分研究及其在烟草中的 应用 $[\mathrm{J}]$ 。食品工业，2015，36(3):115-117。 